\title{
Two ‘Candidatus Liberibacter asiaticus' Strains Recently Found in California Harbor Different Prophages
}

\author{
Z. Zheng, F. Wu, L. B. Kumagai, M. Polek, X. Deng, and J. Chen
}

First, second, and fifth authors: Department of Plant Pathology, South China Agricultural University, Guangzhou, Guangdong, China; third author: Plant Pest Diagnostic Center, California Department of Food and Agriculture, Sacramento; fourth author: U.S. Department of AgricultureAgriculture Research Services, National Clonal Germplasm Repository for Citrus and Dates, Riverside; and sixth author: San Joaquín Valley Agricultural Sciences Center, Parlier, CA.

Accepted for publication 11 February 2017

\begin{abstract}
'Candidatus Liberibacter asiaticus' (CLas), an $\alpha$-proteobacterium, is associated with citrus Huanglongbing (HLB; yellow shoot disease). In California, two cases of CLas have been detected in Los Angeles County, one in Hacienda Heights in 2012 and the other in San Gabriel in 2015. Although all infected trees were destroyed in compliance with a state mandate, citrus industry stakeholder concerns about HLB in California are high. Little is known about the biology of CLas, particularly the California strains, hindering effective HLB management efforts. In this study, next-generation sequencing technology (Illumina MiSeq) was employed to characterize the California CLas strains. Data sets containing $>4$ billion (Giga) bp of sequence were generated from each CLas sample. Two prophages (P-HHCA1-2 and P-SGCA5-1) were identified by the MiSeq read mapping technique referenced to two known Florida CLas prophage sequences, SC1 and SC2. P-HHCA1-2 was an SC2-like or Type 2

prophage of 38,989 bp in size. P-SGCA5-1 was an SC1-like or Type 1 prophage of $37,487 \mathrm{bp}$ in size. Phylogenetic analysis revealed that P-HHCA1-2 was part of an Asiatic lineage within the Type 2 prophage group. Similarly, P-SGCA5-1 was part of an Asiatic lineage within Type 1 prophage group. The Asiatic relatedness of both P-HHCA1-2 and P-SGCA5-1 was further presented by single nucleotide polymorphism analysis at terL (encoding prophage terminase) that has been established for CLas strain differentiation. The presence of different prophages suggests that the two California CLas strains could have been introduced from different sources. An alternative explanation is that there was a mixed CLas population containing the two types of prophages, and limited sampling in a geographic region may not accurately depict the true CLas diversity. More accurate pathway analysis may be achieved by including more strains collected from the regions.
\end{abstract}

'Candidatus Liberibacter asiaticus' (CLas), an alphaproteobacterium (Garnier 2005; Jagoueix et al. 1994), is associated with citrus Huanglongbing (HLB; yellow shoot disease, also known as citrus greening disease). HLB is currently threatening citrus production worldwide (Bove 2006). In the United States, CLas was first detected in a symptomatic citrus tree in Florida in 2005 (Halbert 2005) and subsequently found in several additional southern and gulf states. In California, the first detection of CLas was from an Asian citrus psyllid (ACP, Diaphorina citri) and then traced back to a residential backyard tree in the community of Hacienda Heights of Los Angeles County in 2012 (Kumagai et al. 2013). Three years later, a second detection of CLas in an ACP and multiple citrus trees was reported in the city of San Gabriel, about 15 miles away from the Hacienda Heights site (Yan et al. 2016). In both cases, citrus trees confirmed as positive for HLB-associated bacteria were quickly removed and destroyed and California officially remains HLB-free. However, public concerns about HLB are high, demanding early detection, efficient and effective HLB management, which requires comprehensive biological knowledge about CLas.

To date, CLas has not been cultured in vitro. The bacterial characterization relies on analyses of DNA extracted from infected host

Corresponding authors: J. Chen; E-mail address: Jianchi.chen@ars.usda.gov, and X. Deng; E-mail address: xldeng@scau.edu.cn

First and second authors contributed equally to the paper.

*The $\boldsymbol{e}$-Xtra logo stands for "electronic extra" and indicates that one supplementary figure is published online.

This article is in the public domain and not copyrightable. It may be freely reprinted with customary crediting of the source. The American Phytopathological Society, 2017. (plant or insect) tissues. Characteristic sequence in the 16S rRNA gene is used for CLas species detection but this method lacks the resolution to differentiate strains from different sources (Deng et al. 2008). The genome of CLas is composed of a chromosomal region and a prophage region (phage DNA integrated into bacterial chromosome). While the chromosomal region is highly conserved, the prophage region is highly variable (Duan et al. 2009; Katoh et al. 2014; Zhang et al. 2011; Zheng et al. 2016; Zhou et al. 2011). A short CLas phage sequence was first described by Villechanoux et al. (1993). Later, a complete prophage was reported in the genome of strain Psy62 from Florida (Duan et al. 2009). Zhang et al. (2011) published two circular phage/prophage genomes: SC1 (40,048 bp) and SC2 $(38,997 \mathrm{bp})$ in strain UF506 maintained in periwinkle. Katoh et al. (2014) reported a Japanese strain of CLas without any prophage sequence. Zheng et al. (2016) performed an analysis of prophage types in 187 CLas strains and revealed that most contained mostly single type of prophage, either SC1-like (Type 1) or SC2-like (Type 2). They also reported a CLas strain that lacked both Type 1 and Type 2 prophages.

Variations among CLas prophages provide an ideal target for differentiation of CLas strains. Tomimura et al. (2009) established three phylogenetic clusters of CLas strains in Southeast Asia based on single nucleotide polymorphisms (SNPs) in a bacteriophage sequence (Villechanoux et al. 1993). There are two alternate/ competing methods for categorizing CLas strains: one relies on variations in single locus, e.g., terL in prophage region (Liu et al. 2011) or trn in chromosome or different prophage region (Chen et al. 2010; Deng et al. 2014), and the other is double locus analysis (Deng et al. 2014). Both methods were successfully used to categorize CLas strains from Brazil, China, and the United States. Also, using double locus analysis, Yan et al. (2016) identified differences between California CLas strains from Hacienda 
Heights and San Gabriel. However, this analysis precluded a full study of the prophage composition of the California CLas strains.

In addition to strain variation studies, prophages are known for their ability to influence bacterial biological behaviors. For CLas, prophages/phages have been speculated to influence CLas culturability (Fleites et al. 2014), the eliciting of host defense responses (Jain et al. 2015), and CRISPR/cas activities (Zheng et al. 2016). To facilitate CLas research in California, the draft whole genome sequences of two CLas strains, one from Hacienda Heights and one from San Gabriel, were acquired and published (Wu et al. 2015; Zheng et al. 2014b). Preliminary analyses showed that both strains had prophage sequences.

In this study, a next-generation sequencing (NGS) technology, called MiSeq, was used to study genomic variations between the two Californian CLas strains. The short sequence reads (about 250 bp each) generated by MiSeq from the two CLas samples were mapped onto two known CLas prophage sequences. The mapping profiles were compared with reveal CLas strain differences. Prophage sequences were then extracted, assembled, and analyzed. The CLas strain from Hacienda Heights was found to contain a Type 2 prophage, whereas CLas strain from San Gabriel contained a Type 1 prophage. Biological and epidemiological implications of the two CLas strains were discussed in light of their prophage variation based on in silico analyses.

\section{MATERIALS AND METHODS}

CLas DNA collections. In 2012, a DNA sample was collected from a CLas-infected backyard tree in the Hacienda Heights community of Los Angeles County, California (Kumagai et al. 2013). This sample was designated as strain HHCA1 in this study. In June 2015, several CLas-infected trees were detected in the city of San Gabriel also in Los Angeles County. Two DNA samples from different citrus trees were selected and designated as SGCA1 and SGCA5, respectively. A DNA sample was obtained from a CLaspositive ACP in a separate citrus tree collected in San Gabriel and was designated as SGCApsyT2. All CLas samples were collected by the California Department of Food and Agriculture (CDFA) following the procedure described previously (Kumagai et al. 2013). CLas DNAs were sent to the U.S. Department of AgricultureAgricultural Research Service (USDA-ARS), San Joaquin Valley Agricultural Sciences Center in Parlier, California, for analyses.

DNA sequencing. Brief reports on DNA sequencing of HHCA1 and SGCA5 have been published (Wu et al. 2015; Zheng et al. 2014b). To alleviate the problem of low CLas titer in plant samples, bacterial DNA was enriched using the NEBNext microbiome DNA enrichment kit (New England BioLabs, Inc., Ipswich, MA). The enriched DNA was then enlarged through multiple displacement amplification using the REPLI-g minikit (Qiagen, Inc., Valencia, CA), which amplified all DNA in an unbiased manner. Genome sequencing was carried out by Illumina MiSeq (Illumina, Inc., San Diego, CA) through a commercial source.

Prophage identification, assembling, annotation, and copy number estimation. The prophage sequences of SC1 (NC_019549) and SC2 (NC_019550) were downloaded from the GenBank database. MiSeq sequence reads of strains HHCA1, SGCA1, SGCA5 and SGCApsyT2 were mapped against the SC1 and SC2 sequences separately using Genomics Workbench software version 7.5 (CLC Bio, Denmark). Consensus sequences were identified and whole prophage genome sequences were assembled. In addition, MiSeq reads were used for de novo assembly using Velvet 1.2.10 (Zerbino and Birney 2008). Prophage contigs were identified through BLAST (Camacho et al. 2009) search with SC1 and SC2 as queries and collected by a Perl script. A final prophage genome was determined by combining both referenced and de novo assemblies, in conjunction with PCR and Sanger's sequencing method when necessary. Annotation was performed by the RAST server (http://rast.nmpdr.org/) (Aziz et al. 2008). To evaluate sequence quality of MiSeq prophage assemblies, partial sequences of terL were extracted, aligned and compared with the published terL sequence HM105498.1 generated by Sanger's sequencing method from a Chinese CLas strain (Liu et al. 2011). To detect the presence of circular phage genome DNA, the linear sequences of SC1 and SC2 in GenBank were reorganized by cutting a 5,000 bp sequence from the $3^{\prime}$ end and placed at the $5^{\prime}$ end to create SC1' and SC2', respectively. MiSeq reads were then mapped to SC1' and SC2'. A circular phage genome was indicated by continuity of read coverage through the cutting points of SC1' or SC2'.

To estimate prophage/phage copy numbers, the average nucleotide coverages from SC1 and SC2 mapping were compared with the average nucleotide coverage from CLas chromosome mapping. The CLas chromosome was represented by the genome sequence of strain psy62 after removing its prophage FP1. The CLC genomic workbench software was used to assist sequence analyses.

Acquiring prophage from published CLas sequences. Three published prophage sequences, SC1, SC2, and FP2 (JF773396.1) were downloaded from the GenBank database. Prophages in other CLas strains were extracted from the published whole genome sequences available in GenBank database. These strains included Psy62 (CP001677.5), gxpsy (CP004005.1), A4 (CP010804.1), FL17 (JWHA00000000.1), HHCA (JMIL00000000.2), YCPsy (LIIM00000000.1), and SGCA5 (LMTO00000000.1). Strain Ishi-1 (AP014595.1) was excluded since it was reported to be void of prophages. SC1 and SC2 sequences were used as guides to identify and retrieve prophage region(s)/contigs from the whole genome

TABLE 1. The general information and MiSeq sequence data of four California strains of 'Candidatus Liberibacter asiaticus' and read mapped data referenced to prophages SC1 and SC2

\begin{tabular}{|c|c|c|c|c|c|c|c|c|c|}
\hline \multirow[b]{2}{*}{ Strain } & \multirow[b]{2}{*}{ Location } & \multirow[b]{2}{*}{ Host } & \multicolumn{3}{|c|}{ MiSeq data } & \multicolumn{4}{|c|}{ Prophage-mapped Reads } \\
\hline & & & Orientation $^{\mathrm{a}}$ & Total bp & Total read & SC1 & $\mathrm{SC} 2$ & $\mathrm{SC} 1 / \mathrm{SC} 2$ & $\mathrm{SC} 2 / \mathrm{SC} 1$ \\
\hline \multirow[t]{3}{*}{ HHCA1 (run 1) } & Hacienda Heights & Lemon & $\mathrm{R} 1$ & $5,114,521,340$ & $22,022,950$ & 333 & 693 & & \\
\hline & & & $\mathrm{R} 2$ & $5,527,972,406$ & $22,022,950$ & 328 & 684 & & \\
\hline & & & $\mathrm{R} 1+\mathrm{R} 2$ & $10,642,493,746$ & $44,045,900$ & 661 & 1,377 & 0.48 & - \\
\hline \multirow[t]{3}{*}{ HHCA1 (run 2) } & Hacienda Heights & Lemon & $\mathrm{R} 1$ & $4,327,382,819$ & $17,240,569$ & 234 & 535 & & \\
\hline & & & $\mathrm{R} 2$ & $4,327,382,819$ & $17,240,569$ & 231 & 527 & & \\
\hline & & & $\mathrm{R} 1+\mathrm{R} 2$ & $19,297,259,384$ & $78,527,038$ & 467 & 1,062 & 0.44 & - \\
\hline \multirow[t]{3}{*}{ SGCA5 } & San Gabriel & Citrus & $\mathrm{R} 1$ & $4,793,119,092$ & $19,096,092$ & 653 & 379 & & \\
\hline & & & $\mathrm{R} 2$ & $4,793,119,092$ & $19,096,092$ & 644 & 378 & & \\
\hline & & & $\mathrm{R} 1+\mathrm{R} 2$ & $9,586,238,184$ & $38,192,184$ & 1,297 & 757 & - & 0.58 \\
\hline \multirow[t]{3}{*}{ SGCA1 } & San Gabriel & Citrus & $\mathrm{R} 1$ & $4,469,645,854$ & $17,807,354$ & 195 & 124 & & \\
\hline & & & $\mathrm{R} 2$ & $4,469,645,854$ & $17,807,384$ & 192 & 122 & & \\
\hline & & & $\mathrm{R} 1+\mathrm{R} 2$ & $8,939,291,708$ & $35,614,738$ & 387 & 246 & - & 0.64 \\
\hline \multirow[t]{3}{*}{ SGCA-psyT2 } & San Gabriel & $\mathrm{ACP}$ & $\mathrm{R} 1$ & $5,128,971,148$ & $20,434,148$ & 97 & 55 & & \\
\hline & & & $\mathrm{R} 2$ & $5,128,971,148$ & $20,434,148$ & 99 & 52 & & \\
\hline & & & $\mathrm{R} 1+\mathrm{R} 2$ & $10,257,942,296$ & $40,868,296$ & 196 & 107 & - & 0.55 \\
\hline
\end{tabular}

${ }^{\mathrm{a}} \mathrm{R} 1$ = forward MiSeq reads; $\mathrm{R} 2$ = reverse MiSeq reads. 
sequences. In the case of multiple contigs from a CLas strain, the contigs were connected arbitrarily based on the gene order in SC1 or $\mathrm{SC} 2$. Prophages were named in the following format: $\mathrm{P}$ (meaning prophage)-CLas strain name-prophage type. For example, P-SGCA5-1 describes a prophage from CLas strain SGCA5 that belongs to Type 1 group.
Nucleotide diversity of CLas prophages. A sliding window of $250 \mathrm{bp}$ with a step size of $25 \mathrm{bp}$ was used to calculate the Pi $(\pi)$ values of each prophage gene using DnaSP v5 software (Librado and Rozas 2009) following the method of Wu et al. (2016). Pi value was defined as the average number of nucleotide differences per site between/among sequences (Nei 1987).

\section{SC1 (40,048 bp) - Type 1}

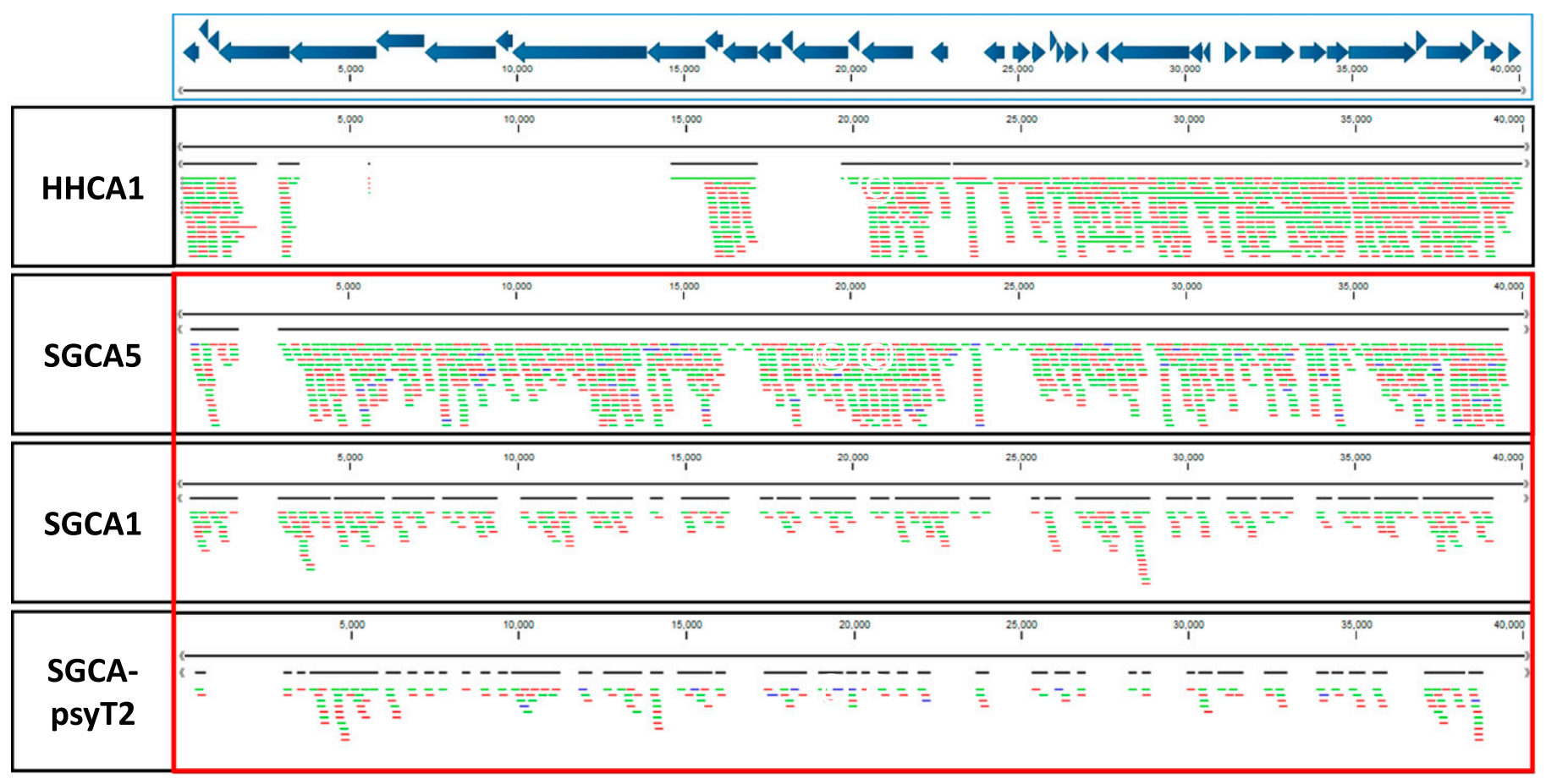

SC2 (38,997 bp) - Type 2

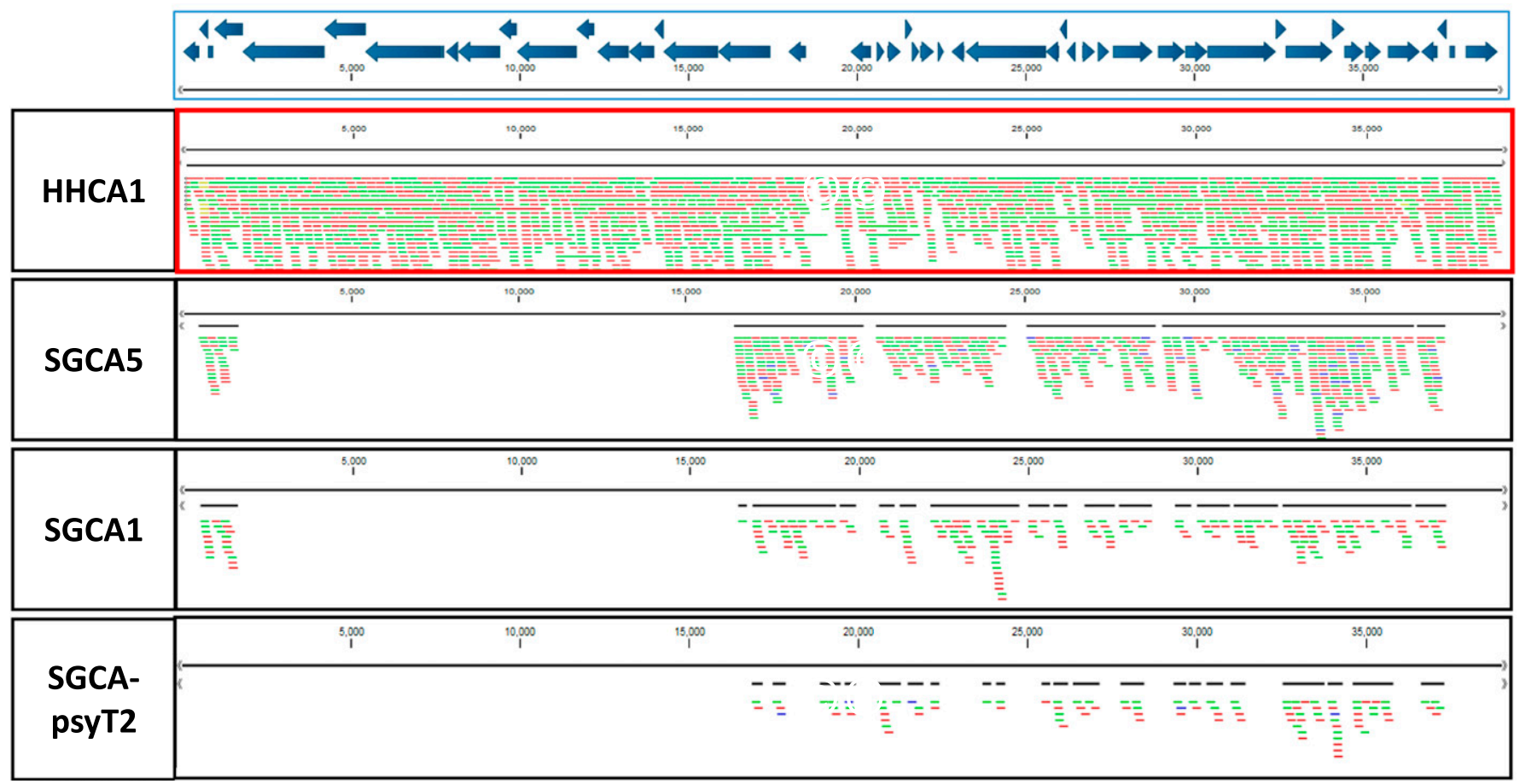

Fig. 1. Mapping track of MiSeq reads ( $250 \mathrm{bp} / \mathrm{read}$, dark gray = forward, light gray = reverse) from 'Candidatus Liberibacter asiaticus' strains collected in Hacienda Heights (HHCA1) and San Gabriel (SGCA5, SGCA1, and SGCA-psyT2), California, referenced to SC1 and SC2 (boxes at top with arrow lines representing open reading frames). Solid black lines above short reads (light and dark gray bars) represent assembled contigs in each strain. Gray boxes position the bacterial strains to the most probable prophage types. Note the presence of a single contig in strain HHCA1 referenced to SC2 and two contigs in strain SGCA5 referenced to SC1. 
Phylogenetic analyses. All prophage sequences were analyzed using Mega (v6.06) software through neighbor-joining distance methods with 1,000 bootstrap replicates (Tamura et al. 2013).

SNP analysis of terL locus. Gene sequences of phage terminase large subunit, terL, in each prophage genome of all published CLas genome sequences were identified based on annotation information and BLAST searches with the specific primer sequences of FC3f/ FC3r and CT3f/CT3r (Deng et al. 2014). Gene sequences were aligned and SNPs in the primer sequences of FC3f/FC3r and CT3f/ CT3r were identified.

\section{RESULTS}

A summary of selected MiSeq data metrics from the four California CLas strains is shown in Table 1. MiSeq sequencing generated over 4 billion (Giga or $\mathrm{G}$ ) bp sequences or 17 to 22 million (Mega or M) sequence reads of $\sim 250 \mathrm{bp} / \mathrm{read}$ for a CLas sample in one direction. Most reads were expected to be of host origin. The numbers of reads that mapped to SC1 and SC2 varied from sample to sample, reflecting the variation in CLas/prophage titers. For a single sample, read numbers between forward (R1) and reverse (R2) were similar, confirming the double sequencing process.

Two MiSeq runs were performed on the HHCA1 sample. A total of 2,439 reads $(1,377+1,062)$ mapped to SC2 with $100 \%$ length coverage (Fig. 1). This generated a consensus of $38,920 \mathrm{bp}$ in a single contig with an average of $15.35 \times$ nucleotide coverage (Table 2 ). The prophage was designated P-HHCA1-2. A circularity test showed the presence of circular P-HHCA1-2. At the terL locus, P-HHCA1-2 was $99.9 \%$ (765/766) similar to HM105498.1 generated by Sanger's sequencing. In contrast, only $1,128(661+467)$ reads were mapped onto SC1 with gaps, the largest gap being over $10,000 \mathrm{bp}$ (Fig. 1). Both run 1 and run 2 yielded SC1/SC2 mapped read ratios of 0.48 and 0.44 , respectively. Therefore, it was concluded that strain HHCA1 had a single Type 2 prophage, P-HHCA1-2, and had no Type 1 prophage.

For strain SGCA5, a total of 1,297 reads were mapped onto SC1 (Table 1) with $94 \%$ length coverage (Fig. 1). This generated two contigs with a total consensus length of 37,487 bp and an $8.12 \times$ average nucleotide coverage (Table 2 ). In contrast, only 757 reads were mapped onto SC2 with gaps, the largest gap being over $15,000 \mathrm{bp}$ (Fig. 1). The ratio of SC2/SC1 was 0.58 . It was, therefore, concluded that strain SGCA5 had a single Type 1 prophage, P-SGCA5-1, and had no Type 2 prophage. For strains SGCA1 and SGCA-psyT2, although the numbers of prophage-mapped reads were significantly smaller than that in SGCA5, the SC2/SC1 ratio remained relatively similar ( 0.64 versus 0.55 , respectively), suggesting the presence of only Type 1 prophage, likely P-SGCA5-1. Nucleotide sequences of the two California CLas prophages were deposited into GenBank with the accession number KX879602 for P-HHCA1-2 and KX879600/KX879601 for P-SGCA5-1.

The copy number analysis between prophage and chromosome was summarized in Table 2. For strain HHCA1, average nucleotide coverage was $15.35 \times$ to the SC2, but 8.03 to the CLas chromosomal region. For reference, consensus size and nucleotide coverage to SC1 mapping were also listed in parentheses (Table 2) and they were significantly smaller, 24,669 and 6.88, respectively. Therefore, HHCA1 was not considered to have SC1-like prophage. The phage/chromosome ratio was 1.91. For strain SGCA5, average nucleotide coverage was $8.12 \times$ to $\mathrm{SC} 1$, but $12.10 \times$ to the chromosomal region. The prophage/chromosome ratio was 0.67. A similar ratio of 0.75 was shown in strain SGCA-psyT4. Yet the prophage/chromosome ratio in strain SGCA1 was 7.7.

The phylogenetic relatedness among the published CLas prophages was presented in Figure 2. P-SGCA5-1 clustered to Type 1 and P-HHCA1-2 clustered to Type 2. Among Type 1 prophages, P-SGCA5-1 separated from SC1 and P-Psy62-1 (FP1), and grouped with the Asiatic Type 1 prophages including P-FL17-1 from central Florida, which probably belonged to the small Asiatic group described earlier (Deng et al. 2014). Among Type 2 prophages, P-HHCA1-2 grouped with the Asiatic/Chinese P-A4-2 prophage and separated from the Floridian SC2 and FP2. Alignments of Asiatic and Floridian specific primer sequences from all CLas prophages were shown in Figure 3. The Asiatic strain specific primers (CT3f/CT3r) matched well with all the prophages of Asiatic origin including P-FL17-1, regardless of prophage type. Similarly, the Florida strain specific primers (FC3f/FC3r) matched all the prophages of Florida origin. In other words, while P-HHCA1-2 and P-SGCA5-1 belonged to different prophage types, they shared a common Asiatic origin.

TABLE 2. Consensus prophage sizes and nucleotide coverage of four California strains of 'Candidatus Liberibacter asiaticus' (CLas) referenced to prophage SC1, $\mathrm{SC} 2$, and chromosomal region (strain Psy62) ${ }^{\mathrm{a}}$

\begin{tabular}{|c|c|c|c|c|c|c|c|}
\hline \multirow[b]{2}{*}{ CLas strain } & \multicolumn{2}{|c|}{$\mathrm{SC} 2(38,997 \mathrm{bp})$} & \multicolumn{2}{|c|}{$\mathrm{SC} 1(40,048 \mathrm{bp})$} & \multicolumn{2}{|c|}{ Chromosomal region $(\sim 1.187 \mathrm{M})$} & \multirow[b]{2}{*}{ Prophage/chromosome } \\
\hline & $\begin{array}{l}\text { Consensus } \\
\text { size (bp) }\end{array}$ & $\begin{array}{c}\text { Average nucleotide } \\
\text { coverage }(x)\end{array}$ & $\begin{array}{l}\text { Consensus } \\
\text { size (bp) }\end{array}$ & $\begin{array}{l}\text { Average nucleotide } \\
\text { coverage }(x)\end{array}$ & $\begin{array}{l}\text { Consensus } \\
\text { size (bp) }\end{array}$ & $\begin{array}{l}\text { Average nucleotide } \\
\text { coverage }(x)\end{array}$ & \\
\hline HHCA1 & 38,920 & 15.35 & $(24,669)$ & $(6.88)$ & $1,056,821$ & 8.03 & 1.91 \\
\hline SGCA1 & $(17,979)$ & (1.48) & 28,656 & 2.31 & $(216,588)$ & 0.30 & 7.7 \\
\hline SGCA-psyT2 & $(10,876)$ & $(0.69)$ & 20,884 & 1.23 & $(755,498)$ & 1.63 & 0.75 \\
\hline
\end{tabular}

a Data in parentheses are not used for analyses.

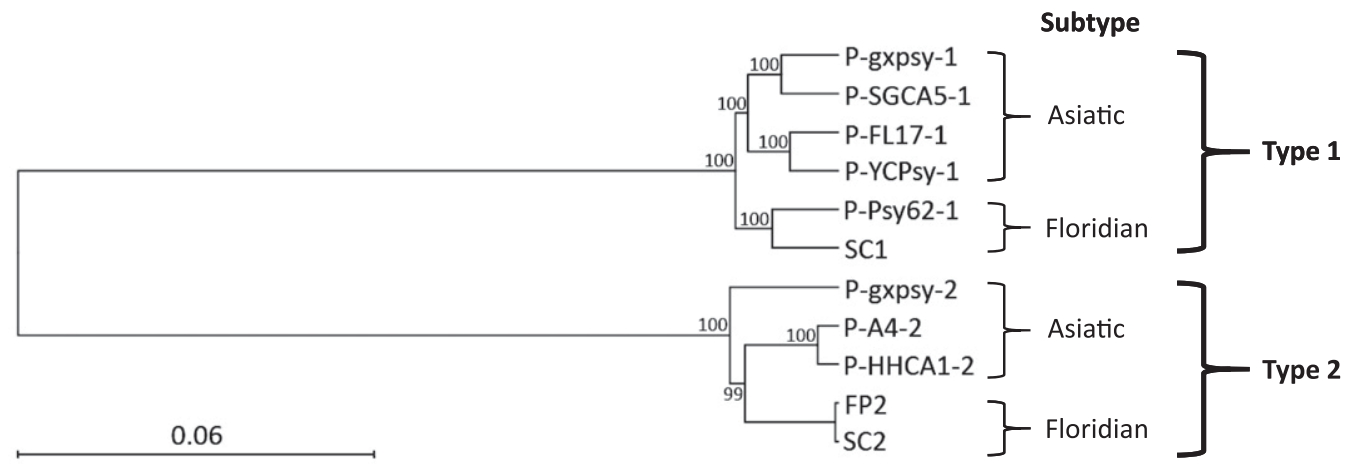

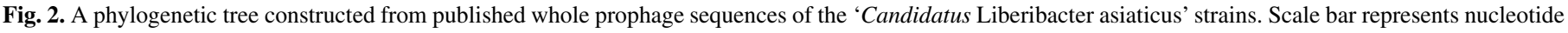
substitution rate. 
Diversity within Type 1 and Type 2 prophages in CLas was further examined by analyzing variations among predicted genes sequences. Nucleotide diversity of predicted genes in the Type 1 prophages (SC1, FP1, P-FL17-1, P-YCPsy-1, P-gxpsy-1, and P-SGCA5-1) is shown in Figure 4A. Similarly, nucleotide diversity among predicted genes in Type 2 prophages (SC2, FP2, P-A4-2, P-gxpsy-2, and P-HHCA-2) is shown in Figure 4B. In both prophage types, there existed significant nucleotide variations among predicted genes. As identified by a box in Figure $4 \mathrm{~A}$ and $\mathrm{B}$, nucleotide diversity of terL among the Type 1 prophages was low (Average $\mathrm{Pi}=0.0066$ ), but relatively high among Type 2 prophages (Average $\mathrm{Pi}=0.0586$ ). However, the $3^{\prime}$ half of the terL, where primer sets CT3f/CT3r and FC3f/FC3r were located, seemed to be conserved among the two prophage types (Fig. 4).

\section{DISCUSSION}

Low CLas titer presents a significant challenge to acquire sufficient DNA sequence depth to cover the complete bacterial genome. An additional challenge is the current quarantine status in California

$\begin{array}{ll}\text { Type } 1 & \\ & \text { P-SGCA5-1 } \\ \text { P-HHCA1-2 } & \text { P-gxpsy-1 } \\ \text { P-gxpsy-2 } & \text { P-A4-2 } \\ & \text { P-FL17-1 } \\ \text { P-YCPsy } & \text { SC1 } \\ \text { SC2 } \\ \text { P-Psy62 } \\ \text { FP2 }\end{array}$

Type 2

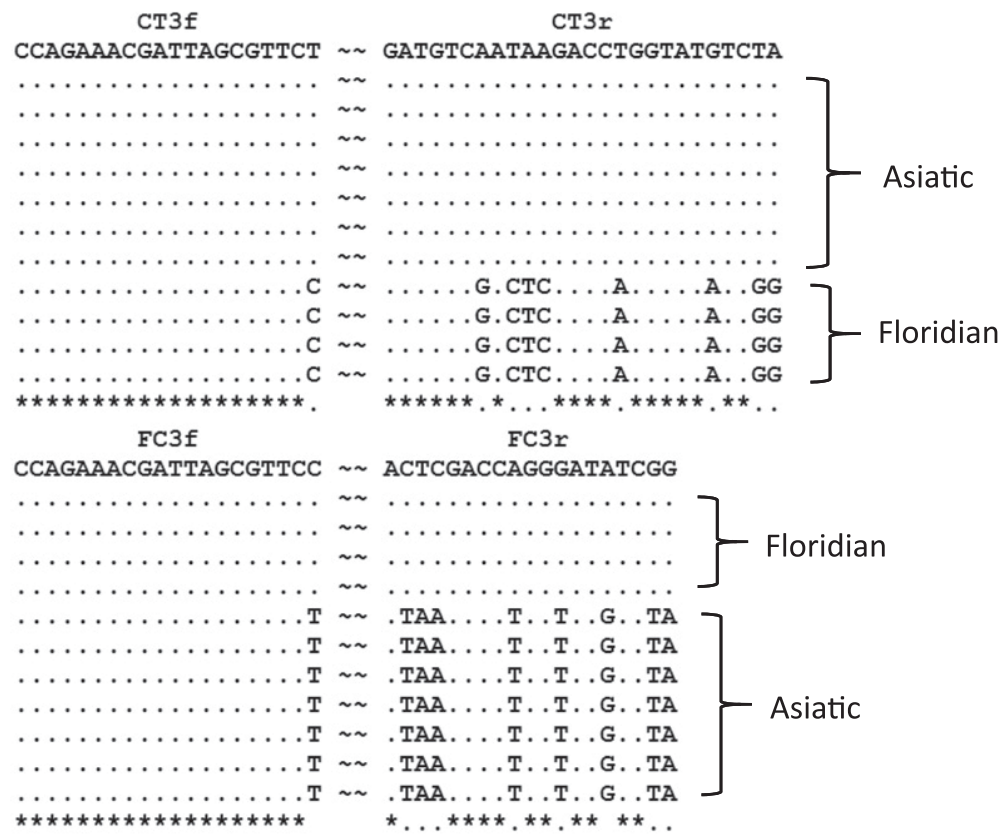

Fig. 3. Alignment and single nucleotide polymorphisms (SNPs) in primer sequences (CT3f and CT3r, Asiatic subtype specific) among Type 1 prophages and in primer sequences (FC3f and FC3r, Floridian subtype specific) among Type 2 prophages. Note that both P-HHCA5-2 and P-SGCA5-1 have no SNPs to CT3f and CT3r.
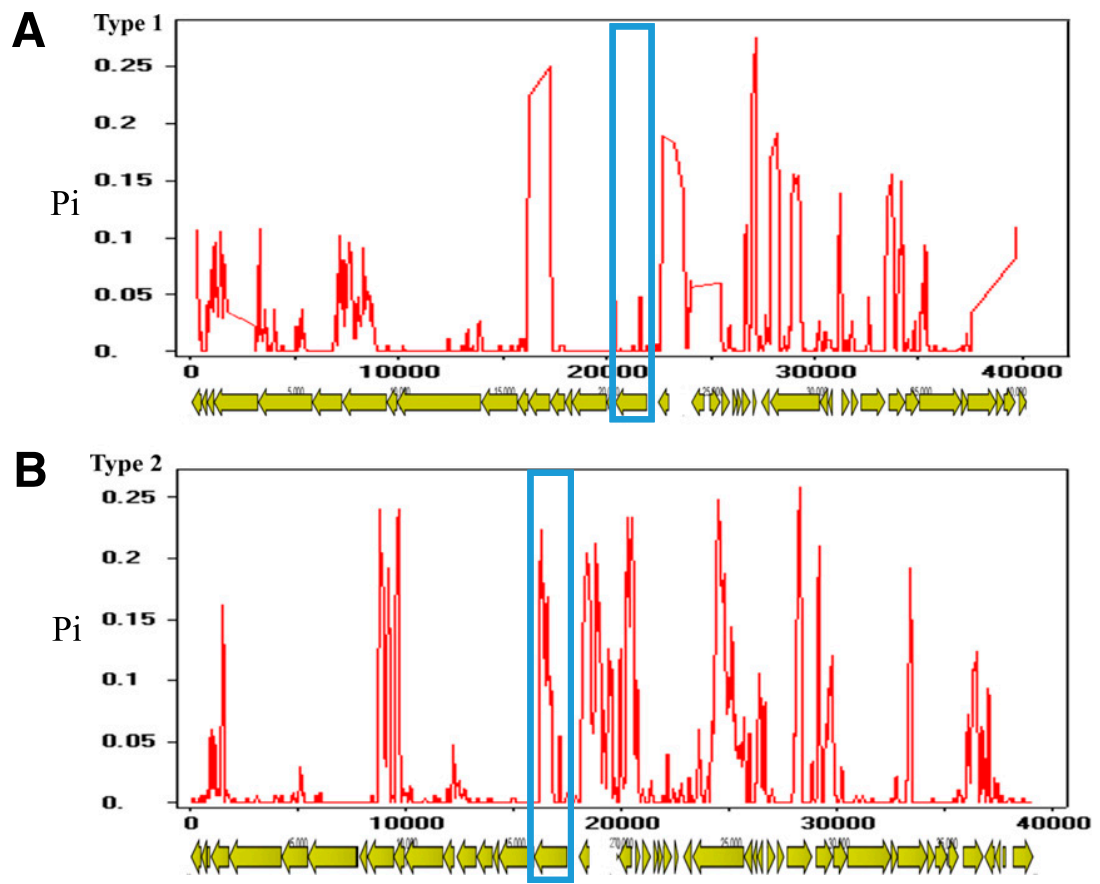

Fig. 4. Nucleotide diversity (Pi) among A, Type 1 and B, Type 2 prophages. A, Sliding windows was computed using SC1, FP1, P-FL17-1, P-YCPsy-1, P-gxpsy-1, and P-SGCA1. Arrow boxes at the bottom are annotation of SC1. B, Sliding windows was computed using SC2, FP2, P-A4-2, P-gxpsy-2, and P-HHCA2. Arrow boxes at the bottom are annotation of SC2. Windows length $=100$ sites, step size $=25$ sites. The terL genes were highlighted in gray box. Note the significant nucleotide diversity at the $5^{\prime}$ half of terL among Type 2 prophages. 
prohibiting the maintenance and propagation of live materials infected with CLas in general research laboratories. For this reason, the amount of CLas sample for research was usually limited to the original volume in DNA extract kit following confirmatory testing procedures $(<50 \mu \mathrm{l})$. With the enrichment and enlargement procedure (Zheng et al. 2014a, b), Giga-bp level DNA sequence data were generated from as little as $2 \mathrm{ng}(0.2 \mathrm{pg} \times 10 \mu \mathrm{l})$ of CLas DNA determined by quantitative PCR (Table 1). Using the MiSeq read mapping technique, two different CLas prophages were identified, P-HHCA1-2 from Hacienda Heights CLas and P-SGCA5-1 from San Gabriel CLas.

Sequence quality of prophages from MiSeq sequencing are comparable, if not better, to that of Sanger's sequencing method. In fact, Supplementary Figure S1 shows that the two MiSeq-based prophage sequences have their nucleotide coverage over $8 \times$ at the terL locus compared with $2 \times$ from Sanger's sequencing method. It should be noted that the goal of this study was to demonstrate difference of CLas strains from two locations in California. Profiles of MiSeq read mapping on prophage region effectively revealed the strain difference (Fig. 1). Prophage sequence analyses further described the bioinformatic variations of the two CLas strains. MiSeq read mapping also provides information to estimate copy numbers of P-HHCA1-2 and P-SGCA5-1. Assuming DNA amplification of CLas genome during enlargement was uniform as stated by the manufacturer, the 1.91 ratio of prophage/chromosome nucleotide coverage can be interpreted as there were two copies of the prophage sequences per CLas cell in the HHCA1 sample. Adding the presence of circular form, P-HHCA1-2 could exist in either two circular plasmid forms or one plasmid form and one linear form in the CLas host.

For the San Gabriel CLas strains, the average nucleotide coverage ratio of P-SGCA5-1 (Type 1 prophage)/chromosome varied from 0.7 (strains SGCA5 and SGCA-psyT2) to 7.7 (SGCA1) (Table 2). In the former, the ratio of 0.7 suggests that only $70 \%$ of the CLas population had prophage, i.e., $30 \%$ did not have prophages. This is not surprising because CLas strains completely void of both Type 1 and Type 2 prophages have been reported (Katoh et al. 2014; Zheng et al. 2016). Based on the same calculation, strain SGCA1 should have eight P-SGCA5-1 copies per CLas cell, likely one as a prophage and seven as circular plasmids. It should be noted that prophage copy numbers are only roughly estimated in this study. More precise estimates need to be determined from control experiments. Nevertheless, this study made the first attempt to quantitatively describe CLas prophages in field collected samples and demonstrated prophage copy number variation. A practical implication from this observation is that for CLas detection using prophage-based primers, prophage variation should be kept in mind for result interpretation.

Based on currently available sequence data, CLas prophages can be grouped into Type 1 and Type 2 (Fig. 3) as reported previously (Zheng et al. 2016). However, as revealed by gene nucleotide diversity (Fig. 4), significant sequence variations exist within each prophage type. Within either Type 1 or Type 2, there exist Floridian and Asiatic subtypes. Two previously published terL-based primer sets (Deng et al. 2014) could be used to differentiate the two subtypes: The American subtypes can be represented by primer set FC3f/FC3r, and the Asiatic subtypes can be represented by primer set CT3f/CT3r (Fig. 3). The discriminatory capacity of the two primer sets was tested and confirmed with >300 CLas samples collected from Florida and China, as well as Brazil, which had the Asiatic subtype at terL locus (Term-A group) (Deng et al. 2014).

Interestingly, both CT3f/CT3r and FC3f/FC3r are located at the conserved $3^{\prime}$ end half of terL (Fig. 4). It can be expected that analysis on the $5^{\prime}$ end portion of ter $L$ could expose additional CLas subtypes. Along this line, other prophage genes are also of value for CLas strain diversity research. Zheng et al. (2016) identified eight Type 1 prophage specific and seven Type 2 prophage specific loci and designed PCR primer sets accordingly for a prophage typing assay. The relationships and utilities between the prophage-type specific loci and geographic-specific loci in CLas prophages deserve further evaluation.
The fact that CLas strains HHCA1 and SGCA5 carry different prophages suggests that they were introduced from separate sources. For San Gabriel samples, MiSeq read mapping showed all three CLas strains (SGCA1, AGCA5, and SGCApsyT4) had the same/highly similar prophage (Fig. 1). Therefore, it is assumed that a single strain of CLas was introduced in the San Gabriel area and the bacterium was subsequently spread by ACP. Such evidence is, however, lacking for the Hacienda Heights strain, because only a single CLas strain was analyzed.

Alternatively, considering the simultaneous presence of both Type 1 and Type 2 CLas prophages in Florida and China, it is possible that a single CLas population containing both Type 1 and Type 2 prophages was introduced in California. Detection of a single prophage in either Hacienda Heights or San Gabriel might be due to the limited number of samples analyzed.

More accurate pathway analysis may be achieved by genomic analysis of multiple strains from California when/if detected.

In summary, with the imminent threat of HLB to the California citrus industry, it is urgent that we have comprehensive knowledge about CLas for successful HLB management. We have overcome some obstacles in CLas research by taking advantage of the new NGS technology and sequence analysis capacity. We characterized two prophages from the CLas strains recently found in California, proposing that the two California CLas strains were of recent Asiatic origin and may have been introduced separately, although more studies about the California CLas population are needed. Absent a breakthrough in successful in vitro cultivation, NGS analysis of more CLas strains in planta remains the most promising means by which to acquire CLas biological information.

\section{ACKNOWLEDGMENTS}

We thank S. Vargas for technical support. This research was supported by research funds from the California Citrus Research Board and Chinese Modern Agricultural Technology Systems (CARS-27). The mention of trade names or commercial products in this publication is solely for the purpose of providing specific information and does not imply recommendation or endorsement by the U.S. Department of Agriculture.

\section{LITERATURE CITED}

Aziz, R. K., Bartels, D., Best, A. A., DeJongh, M., Disz, T., Edwards, R. A., Formsma, K., Gerdes, S., Glass, E. M., Kubal, M., Meyer, F., Olsen, G. J., Olson, R., Osterman, A. L., Overbeek, R. A., McNeil, L. K., Paarmann, D., Paczian, T., Parrello, B., Pusch, G. D., Reich, C., Stevens, R., Vassieva, O., Vonstein, V., Wilke, A., and Zagnitko, O. 2008. The RAST server: Rapid annotations using subsystems technology. BMC Genomics 9:75.

Bove, J. M. 2006. Huanglongbing: A destructive, newly-emerging, century-old disease of citrus. J. Plant Pathol. 88:7-37.

Camacho, C., Coulouris, G., Avagyan, V., Ma, N., Papadopoulos, J., Bealer, K., and Madden, T. L. 2009. BLAST+: Architecture and applications. BMC Bioinform. 10:421.

Chen, J., Deng, X., Sun, X., Jones, D., Irey, M., and Civerolo, E. 2010. Guangdong and Florida populations of 'Candidatus Liberibacter asiaticus' distinguished by a genomic locus with short tandem repeats. Phytopathology 100:567-572.

Deng, X., Chen, J., Feng, Z., Shan, Z., Guo, H., Zhu, J., Li, H., and Civerolo, E. 2008. Identification and characterization of the Huanglongbing bacterium in pummelo from multiple locations in Guangdong, P.R. China. Plant Dis. 92:513-518.

Deng, X., Lopes, S., Wang, X., Sun, X., Jones, D., Irey, M., Civerolo, E., and Chen, J. 2014. Characterization of 'Candidatus Liberibacter asiaticus' populations by double-locus analyses. Curr. Microbiol. 69:554-560.

Duan, Y., Zhou, L., Hall, D. G., Li, W., Doddapaneni, H., Lin, H., Liu, L., Vahling, C. M., Gabriel, D. W., Williams, K. P., Dickerman, A., Sun, Y., and Gottwald, T. 2009. Complete genome sequence of citrus huanglongbing bacterium 'Candidatus Liberibacter asiaticus' obtained through metagenomics. Mol. Plant-Microbe Interact. 22:1011-1020.

Fleites, L. A., Jain, M., Zhang, S., and Gabriel, D. W. 2014. 'Candidatus Liberibacter asiaticus' prophage late genes may limit host range and culturability. Appl. Environ. Microbiol. 80:6023-6030.

Garnier, M. 2005. Genus V. Candidatus Liberibacter Jagoueix, Bove' and Garnier 1994, 385 (Candidatus Liberobacter (sic) Jagoueix, Bove' and 
Garnier 1994, 385). Pages 400-402 in: Bergey’s Manual of Systematic Bacteriology, 2nd ed. Vol. 2, The Proteobacteria, Part C. The Alpha-, Beta-, Delta-, and Epsilon-Proteobacteria. D. J. Brenner, N. R.Krieg, and J. T. Staley, eds. Springer, New York.

Halbert, S. E. 2005. The discovery of Huanglongbing in Florida. Proceedings of 2nd International Citrus Canker and Huanglongbing Research Workshop H-3, Florida Citrus Mutual, Orlando, FL.

Jagoueix, S., Bové, J. M., and Garnier, M. 1994. The phloem-limited bacterium of greening disease of citrus is a member of the alpha subdivision of the Proteobacteria. Int. J. Syst. Bacteriol. 44:379-386.

Jain, M., Fleites, L., and Gabriel, D. W. 2015. Prophage encoded peroxidase in 'Candidatus Liberibacter asiaticus' is a secreted effector that suppresses plant defenses. Mol. Plant-Microbe Interact. 105:1043-1049.

Katoh, H., Miyata, S., Inoue, H., and Iwanami, T. 2014. Unique features of a Japanese 'Candidatus Liberibacter asiaticus' strain revealed by whole genome sequencing. PLoS One 9:e106109.

Kumagai, L., Levesque, C., Blomquist, C. L., Madishetty, K., Guo, Y. Y., Woods, P., Rooney-Latham, S., Rascoe, J., Gallindo, T., Schnabel, D., and Polek, M. 2013. First report of 'Candidatus Liberibacter asiaticus' associated with citrus Huanglongbing (HLB) in California. Plant Dis. 97:283.

Librado, P., and Rozas, J. 2009. DnaSP v5: A software for comprehensive analysis of DNA polymorphism data. Bioinformatics 25:1451-1452.

Liu, R., Zhang, P., Pu, X., Xing, X., Chen, J., and Deng, X. 2011. Analysis of a prophage gene frequency revealed population variation of 'Candidatus Liberibacter asiaticus' from two citrus-growing provinces in China. Plant Dis. 95:431-435.

Nei, M. 1987. Molecular Evolutionary Genetics. Columbia University Press, New York.

Tamura, K., Stecher, G., Peterson, D., Filipski, A., and Kumar, S. 2013. MEGA6: Molecular evolutionary genetics analysis version 6.0. Mol. Biol. Evol. 30:2725-2729.

Tomimura, K., Miyata, S. I., Furuya, N., Kubota, K., Okuda, M., Subandiyah, S., Hung, T. H., Su, H. J., and Iwanami, T. 2009. Evaluation of genetic diversity among 'Candidatus Liberibacter asiaticus' isolates collected in Southeast Asia. Phytopathology 99:1062-1069.
Villechanoux, S., Garnier, M., Laigret, F., Renaudin, J., and Bové, J. M. 1993. The genome of the non-cultured, bacterial-like organism associated with citrus greening disease contains the nusGrplKAJL-rpoBC gene cluster and the gene for a bacteriophage type DNA polymerase. Curr. Microbiol. 26:161-166.

Wu, F., Cen, Y., Wallis, C. M., Trumble, J. T., Prager, S., Yokomi, R., Zheng, Z., Deng, X., Chen, J., and Liang, G. 2016. The complete mitochondrial genome sequence of Bactericera cockerelli and comparison with three other Psylloidea species. PLoS One 11:e0155318.

Wu, F., Kumagai, L., Liang, G., Deng, X., Zheng, Z., Keremane, M., and Chen, J. 2015. Draft genome sequence of 'Candidatus Liberibacter asiaticus' from a citrus tree in San Gabriel, California. Genome Announc. 3:e01508-15.

Yan, Z., Rascoe, J., Kumagai, L.B., Keremane, M. L., and Nakhla, M. K. 2016. Citrus Huanglongbing (HLB) discoveries in California in 2015 and 2012 are of different genotypes of Candidatus Liberibacter asiaticus (CLas) by double-locus genomic variation analysis. Plant Dis. 100:645.

Zerbino, D. R., and Birney, E. 2008. Velvet: Algorithms for de novo short read assembly using de Bruijn graphs. Genome Res. 18:821-829.

Zhang, S., Flores-Cruz, Z., Zhou, L., Kang, B. H., Fleites, L., Gooch, M. D., Wulff, N. A., Davis, M. J., Duan, Y., and Gabriel, D. W. 2011. 'Ca. Liberibacter asiaticus' carries an excision plasmid prophage and a chromosomally integrated prophage that becomes lytic in plant infections. Mol. Plant-Microbe Interact. 24:458-468.

Zheng, Z., Bao, M., Wu, F., Chen, J., and Deng, X. 2016. Predominance of single prophage carrying a CRISPR/cas system in 'Candidatus Liberibacter asiaticus' strains in southern China. PLoS One 11:e0146422.

Zheng, Z., Deng, X., and Chen, J. 2014a. Whole-genome sequence of 'Candidatus Liberibacter asiaticus' from Guangdong, China. Genome Announc. 2:e00273-14.

Zheng, Z., Deng, X., and Chen, J. 2014b. Draft genome sequence of 'Candidatus Liberibacter asiaticus' from California. Genome Announc. 2:e00999-14.

Zhou, L., Powell, C. A., Hoffman, M. T., Li, W., Fan, G., Liu, B., Lin, H., and Duan, Y. 2011. Diversity and plasticity of the intracellular plant pathogen and insect symbiont 'Candidatus Liberibacter asiaticus' as revealed by hypervariable prophage genes with intragenic tandem repeats. Appl. Environ. Microbiol. 77:6663-6673. 